\title{
CUATRO CIGARRILLAS DE LA SUBFAMILIA TYPHLOCYBINAE (HOMOPTERA, CICADELLIDAE) NUEVAS PARA LA FAUNA ESPAÑOLA
}

\author{
D. Aguin Pombo (*)
}

\begin{abstract}
RESUMEN
Los Tiflocíbinos son un grupo de insectos fitófagos poco conocidos en la Península Ibérica. Hasta ahora han sido citadas solamente unas setenta especies de cigarrillas. Del estudio del material recogido principalmente en el noroeste de España han resultado cuatro cigarrillas nuevas para la fauna española pertenecientes a la tribu Typhlocibini. De estas, dos especies son nuevas para la Península: Wagneripteryx germari (Zetterstedt, 1840) y Edwardsiana hippocastani (Edwards, 1888); las otras dos: Eurhadina ribauti Wagner, 1935 y Eupteryx filicum (Newman, 1853), son dos citas nuevas para España. Para cada especie se incluye información sobre su distribución, biología y plantas nutricias.
\end{abstract}

Palabras clave: Homoptera, Cicadellidae, cigarrillas, distribución.

\section{ABSTRACT}

\section{Four leafhoppers of the subfamily Typhlocybinae (Homoptera, Cicadellidae) new to the Spanish fauna}

Typhlocibid leafhoppers are a group of phytophagous insects little known in the Iberian Peninsula. Until now only about seventy leafhopper species have been recorded. After the study of material collected mainly from the northwest of Spain, four new species records belonging to the tribe Typhlocibini were found. Of those, two species are new to the Iberian Peninsula: Wagneripteryx germari (Zetterstedt, 1840) and Edwardsiana hippocastani (Edwards, 1888), the other two: Eupteryx filicum (Newman, 1853) and Eurhadina ribauti Wagner, 1935, are new records to Spain. For each species information on distribution, biology and food plants is included.

Key words: Homoptera, Cicadellidae, leafhoppers, distribution.

\section{Introducción}

Los Tiflocíbidos son insectos fitófagos de pequeñas dimensiones que se encuentran asociados a numerosas especies de plantas, que componen tanto el estrato arbóreo, herbáceo como arbustivo (Hamilton, 1985; Le Quesne et al., 1981; Ossiannilsson, 1981). El grado de especificidad que presentan por las plantas de las que se alimentan varía, y según el número de plantas nutricias utilizadas, pueden ser reconocidas especies monófagas, oligófagas o polífagas (Claridge et al., 1976, 1981).

La distribución de los Tiflocíbinos en Europa es relativamente bien conocida (Drosopoulos et al., 1986; D’Urso, 1995; Nast, 1972, 1987), a excepción de la Península Ibérica, donde el número de especies citadas es todavía menos de la mitad de las que se conocen en otras regiones europeas de

* Departamento de Biologia, Universidade de Madeira, Campus da Penteada, 9000 Funchal, Madeira, Portugal. aguin@uma.pt 
dimensiones similares. La escasez de datos, especialmente sobre España, es una laguna importante para conocer la distribución de las especies de esta subfamilia, y también un obstáculo para la realización de estudios de carácter ecológico, agrícola y/o evolutivo.

De los pocos trabajos detallados sobre cigarrillas realizados en España, una parte importante trata precisamente de especies que constituyen plagas y causan enfermedades a cultivos de diversas hortícolas (Medina et al., 1982; Ruiz Castro, 1943), a frutales (Hermoso de Mendoza et al., 1979; Llacer et al., 1986; Medina et al., 1981) y a la vid (Ruiz Castro, 1943; Ruiz Castro et al.,1939). Desde el punto de vista fitosanitario, los daños causados por las cigarrillas a las plantas pueden ser básicamente de dos tipos. En primer lugar, destacan los efectos cloróticos de las hojas (Llacer et al., 1986; Ruiz Castro et al.,1939) que pueden ocasionar caída de las mismas y como consequencia de ello, pérdida de producción. En segundo lugar, destacan los daños producidos por los virus y micoplasmas (Della Giustina, 1989) que son transmitidos por estos insectos a las plantas que hospedan.

\section{Material y métodos}

A excepción de los especímenes del género Wagneripteryx, propiedad del Museo de Ciencias Naturales de Madrid (MNCM), todo el material estudiado ha sido recogido en la provincia de Pontevedra entre los años 1991 y 1999, mediante mangueo sobre la vegetación con una red entomológica. A los machos estudiados, les ha sido retirada la cápsula genital, para ser hervida durante unos minutos en una solución de hidróxido de potasio $(\mathrm{KOH})$ al $10 \%$. Posteriormente, la genitalia ha sido lavada con agua destilada y colocada en una gota de glicerina sobre una lámina portaobjetos para ser posteriormente observada a la lupa.

\section{Resultados}

En total han sido encontradas cuatro especies nuevas para España. Muchas de las especies de esta subfamilia utilizan un número restringido de plantas nutricias, siendo en muchos casos esta información imprescindible para poder identificarlas (Ribaut, 1936; Vidano et al., 1987). Debido a esto, en este trabajo ha sido recopilada para cada especie una lista de las plantas nutricias además de otros datos sobre su biología y distribución. La informa- ción sobre su distribución en la región Paleártica se ha agrupado por áreas manteniendo el criterio usado por Nast (1972) y para las plantas nutricias (Tabla 1) se ha seguido la nomenclatura utilizada en la Flora Europaea (Tutin et al., 1964, 1968, 1980). En la determinación del material se han utilizado los trabajos de Ribaut (1936) y Ossiannilsson (1981) que poseen ilustraciones detalladas de las estruturas de la genitalia de estas especies.

\section{Eurhadina ribauti Wagner, 1935}

Material eXAminado: 4225'N 847'O, 17.VIII.1994, Montalvo, Portonovo (Pontevedra), $20 \mathrm{~m}, 10^{\circ}$, sobre Castanea sativa Mill.

Esta especie es una nueva cita para España y ha sido muestreada en castaños aislados en un pinar situado al lado de campos de cultivo. Es polífaga, se alimenta de varias especies de árboles de hoja caduca pertenecientes a las familias: Tiliaceae, Ulmaceae, Betulaceae y Fagaceae (Claridge et al., 1976, 1981; Augusto Mendez, 1959; Le Quesne et al., 1981; Ribaut, 1936) (Tabla 1). En Gales, Quercus robur y $Q$. petraea, son sus plantas huéspedes (Claridge et al., 1981). Es una especie univoltina que pasa el invierno en estado de huevo (Remane, 1987) y los adultos aparecen de julio a septiembre (Ossiannilsson, 1981).

Se distribuye al oeste de la región Paleártica desde la Península Ibérica hasta el sudeste asiático (Dlabola, 1981; Drosopoulos et al., 1986; Nast, 1972, 1987). En Europa ha sido citada para: Alemania, Bélgica, Chipre, Dinamarca, Finlandia, Francia, Gran Bretaña, Grecia, Holanda, Noruega, Suecia, Suiza y antigua Yugoslavia.

\section{Wagneripteryx germari (Zetterstedt, 1840)}

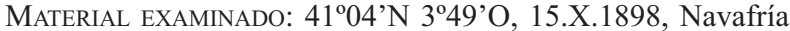
(Madrid), 10", 3 우 우 .

Esta es una especie monófaga que se encuentra asociada a varias especies de pinos (Jankovic, 1984; Günthart et al., 1981; Remane, 1987) (Tabla 1). Es univoltina (Günthart et al., 1981), pasa el invierno en estado de huevo (Remane, 1987) y los adultos están presentes de junio a octubre (Ossiannilsson, 1981).

Es la primera cita de esta especie para la Península Ibérica. Tiene distribución eurosiberiana (Drosopoulos et al. 1986; D'Urso et al., 1990; Nast, 1972, 1987) pero también se encuentra en el norte 
Tabla 1. - Plantas nutricias referidas en la bibliografía para las cuatro especies de cigarrillas. Las plantas en las que desarrollan todo el ciclo biológico están indicadas por un asterisco.

Bibliografía (References): 1= Augusto Mendes, 1959; 2= Claridge et al., 1976; 3= Claridge et al., 1981; 4= Della Giustina, 1989; 5= D’Urso et al., 1990; 6= Günthart et al. 1981, 7= Günthart, 1987; 8= Hamilton, 1985; 9= Jankovic, 1984; 10= Le Quesne et al., 1981; 11= Lindberg, 1961; 12= Ossiannilsson, 1981; 13= Ottosson et al., 1983; 14= Quartau et al., 1988; 15= Remane, 1987; 16= Ribaut, 1936; 17= Schiemenz, 1990; 18= Stewart, 1988; 19= Vidano et al., 1987; $20=$ Vidano y Arzone, 1987a; 21= Vidano y Arzone, 1987b.

Table 1.- Food plants reported in the scientific literature for the four leafhopper species. The plants in which they develop all their biological cycle are indicated by an asterisk.

\begin{tabular}{|c|c|c|c|c|}
\hline Especies & Especificidad & Familias de plantas & Especies de plantas & Bibliografía \\
\hline \multirow[t]{9}{*}{ Eupteryx filicum } & \multirow[t]{9}{*}{ Polífaga en helechos } & ASPLENIACEAE & Phyllitis scolopendrium * & 18 \\
\hline & & \multirow[t]{4}{*}{ ASPIDIACEAE } & Polystichum setiferum * & 18 \\
\hline & & & Dryopteris borreri & 13 \\
\hline & & & Dryopteris dilatata & 13 \\
\hline & & & Dryopteris filix-mas * & 18 \\
\hline & & \multirow[t]{2}{*}{ PolypodiaceAE } & Polypodium vulgare * & 10,18 \\
\hline & & & Polypodium interjectum * & 18 \\
\hline & & ATHYRIACEAE & Athyrium sp. & 19 \\
\hline & & HYPOLEPIDACEAE & Pteridium aquilinum & 11,14 \\
\hline \multirow[t]{6}{*}{ Eurhadina ribauti } & \multirow[t]{6}{*}{ Polífaga en árboles caducifolios } & \multirow[t]{3}{*}{ FAGACEAE } & Quercus robur * & 3 \\
\hline & & & Quercus petraea* & 3 \\
\hline & & & Castanea sativa & 1 \\
\hline & & ULMACEAE & Ulmus sp. & 10 \\
\hline & & Betulaceae & Alnus glutinosa & 2,16 \\
\hline & & TILIACEAE & Tilia sp. & 12,19 \\
\hline \multirow[t]{21}{*}{ Edwardsiana hippocastani } & \multirow[t]{21}{*}{ Polífaga en árboles caducifolios } & \multirow[t]{4}{*}{ BetULACEAE } & Betula pubescens & 2 \\
\hline & & & Betula pendula & 4 \\
\hline & & & Alnus glutinosa * & $2,3,21$ \\
\hline & & & Alnus incana & 21 \\
\hline & & \multirow[t]{5}{*}{ FAGACEAE } & Castanea sativa & 20 \\
\hline & & & Quercus robur & 2,20 \\
\hline & & & Quercus cerris & 20 \\
\hline & & & Quercus petraea & 20 \\
\hline & & & Fagus sp. & 17 \\
\hline & & \multirow[t]{2}{*}{ CoRYLACEAE } & Corylus avellana * & $1,2,3$ \\
\hline & & & Carpinus sp. & 17 \\
\hline & & ACERACEAE & Acer pseudoplatanus & 3 \\
\hline & & \multirow[t]{2}{*}{ ULMACEAE } & Ulmus glabra * & 2,3 \\
\hline & & & Ulmus procera & 3 \\
\hline & & HIPPOCASTANACEAE & Aesculus hippocastanum & 8 \\
\hline & & TILIACEAE & Tilia sp. & 7 \\
\hline & & JUNGLANDACEAE & Junglans sp. & 7 \\
\hline & & SALICACEAE & Salix sp. & 7 \\
\hline & & TAMARICACEAE & Tamarix sp. & 4 \\
\hline & & \multirow[t]{2}{*}{ ROSACEAE } & Sorbus aucuparia & 2 \\
\hline & & & Malus sp. & 7 \\
\hline \multirow[t]{5}{*}{ Wagneripteryx germari } & \multirow[t]{5}{*}{ Monófaga en pinos } & \multirow{5}{*}{ PINACEAE } & Pinus sylvestris & $3,6,9,12,15$ \\
\hline & & & Pinus nigra subsp. laricio & 5,6 \\
\hline & & & Pinus mugo & 6,15 \\
\hline & & & Pinus peuce & 15 \\
\hline & & & Pinus cembra & 6 \\
\hline
\end{tabular}

de África (Le Quesne et al., 1981). En Europa se conoce en: Alemania, Austria, Bélgica, Bulgaria, República Checa, Dinamarca, Eslovaquia, Estonia, Finlandia, Francia, Gran Bretaña, Grecia, Holanda, Irlanda, Italia (y Sicilia), Letonia, Lituania, Noruega, Polonia, Rusia, Suecia, Suiza, Ucrania y antigua Yugoslavia.

\section{Eupteryx filicum (Newman, 1853)}

MATERIAL EXAMINADO: $42^{\circ} 25^{\prime} \mathrm{N} \quad 8^{\circ} 47^{\prime} \mathrm{O}, \quad 3 . I X .1991$ y 5.IX.1991, Portonovo (Pontevedra), $5 \mathrm{~m}, 10^{x}$ y 2 우 우 respectivamente, sobre Pteridium aquilinum (L.) Kuhn.

Es una especie propia de Pteridofitos (Stewart, 1988; Ottosson et al., 1983; Lindberg, 1961; Quar- 
tau et al., 1988), que se encuentra en el centro y sur de Europa (Bélgica, Bulgaria, República Checa, Francia, Gran Bretaña, Grecia, Holanda, Italia, Irlanda, Polonia, Portugal, Suiza y antigua Yugoslavia) incluyendo la mayor parte de las islas atlánticas más próximas (Archipiélagos de Madeira, Azores y Canarias) (D'Urso et al., 1990; Nast, 1972, 1987). En Gales tiene dos generaciones anuales (Stewart, 1988).

\section{Edwardsiana hippocastani (Edwards, 1888)}

Material eXAminado: $42^{\circ} 25^{\prime} \mathrm{N} 8^{\circ} 47^{\prime} \mathrm{O}$, 5.IX.1999, Portonovo (Pontevedra), 5m, $1 \sigma^{7}$ sobre Castanea sativa.

Aunque los especímenes estudiados han sido encontrados unicamente sobre cataños, las plantas nutrícias recopiladas en la bibliografía muestran que es una especie polífaga en árboles de hoja caduca (Vidano y Arzone, 1987a, 1987b; Günthart, 1987; Schiemenz, 1990) (Tabla 1). Probablemente tiene dos generaciones al año y los adultos se encuentran entre los meses de junio y octubre (Ossiannilsson, 1981). Su distribución es holoártica; se encuentra por toda Europa hasta el Irán y en la región Neártica está en Canada (Dlabola, 1981; Drosopoulos et al. 1986; Hamilton, 1985; Nast, 1972, 1987; Vidano y Arzone, 1987a). En Europa se conoce en el archipiélago de Madeira, Alemania, Austria, Bélgica, República Checa, Dinamarca, Eslovaquia, Finlandia, Francia, Gran Bretaña, Grecia, Holanda, Italia, Lituania, Moldavia, Noruega, Polonia, Suecia, Suiza y Ucrania.

\section{AGRADECIMIENTOS}

Quiero agradecer a la conservadora del MNCM, Isabel Izquierdo, por haberme cedido parte del material de este estudio y a J.M. Nieto Nafría por las sugerencias hechas durante la revisión de este trabajo.

\section{Bibliografía}

Augusto Mendes, M., 1959. A entomofauna do castanheiro (Castanea sativa Miller) no concelho de Moimenta da Beira. Publicações da Direcção Geral dos Serviços Florestais e Agrícolas, 26:126-275.

Claridge, M.F. \& Wilson, M.R., 1976. Diversity and distribution patterns of some mesophyll-feeding leafhoppers of temperate woodland canopy. Ecological Entomology, 1: 231-250.

Claridge, M.F. \& Wilson, M.R., 1981. Host plant associations, diversity and species-area relationships of mesophyll-feeding leafhoppers of trees and shrubs in Britain. Ecological Entomology, 6: 217-238.

Della Giustina, W., 1989. Homoptères Cicadellidae 3. Compléments aux ouvrages d'Henri Ribaut. Faune de France 73. INRA. París. 350 pp.

Dlabola, J., 1981. Ergebnisse der tschechoslowkishIranischen entomologischen expeditionen nach dem Iran (1970 und 1973). Acta Entomologica Musei Nationalis Pragae, 40: 127-311.

Drosopoulos, S., Asche, M. \& Hoch, H., 1986. A preliminary list and some notes on the Cicadomorpha (Homoptera-Auchenorrhyncha) collected in Greece. Proceedings of the $2^{\text {nd }}$ International Congress of Rhynchota Balkan Mikrolimmi, Grecia. 8-13.

D’Unso, V., 1995. Homoptera Auchenorrhyncha. In: Minelli, A., Ruffo, S. \& La Posta, S., (eds.). Checklist delle species della fauna italiana 42. Calderini, Bologna: 35 pp.

D’Urso, V. \& Guglielmino, A., 1990. Cicadellidae of the forests of Etna (Hemiptera, Homoptera, Auchenorrhyncha). Scopolia, supl., 1: 77-87.

GüNTHART, H., 1987. Oekologische Untersuchungen im Unterengadin. Zikaden (Auchenorrhyncha). Ergebnisse der wissenschaftlichen Untersuchungen im Schweizerischen Nationalpark, 12: 203-299.

GÜNTHART, H. \& GÜNTHART, M., 1981. Biology and feeding behaviour of Auguriahana germari (Zett.) (Homoptera, Auchenorrhyncha, Typhlocybinae). Proceedings of the $4^{\text {th }}$ Auchenorrhyncha meeting. Finland. 24.

Hamilton, K.G.A., 1985. Cicadelles des arbres ornamentaux et frutiers du Canada. Agriculture Canada, Publicación 1779/E. 71 pp.

Hermoso de Mendoza, A. \& Medina, V., 1979. Estudio inicial sobre cicadélidos (Homoptera, Cicadellidae) en los huertos de agrios del país valenciano. Anales del INIA. Serie Protección Vegetal, 10: 43-68.

JANKovic, L., 1984. Homoptera Auchenorrhyncha (Insecta). Fauna Durmitoria, 1: 229-282.

Llácer, G., Medina, V. \& Archelos, D., 1986. Investigaciones sobre la detección, difusión natural y control del enrrollamiento clorótico del albaricoquero. Boletín de Sanidad Vegetal. Plagas, 12:181-207.

Le Quesne, W.J. \& Payne, K.R., 1981. Cicadellidae (Typhlocybinae) with a check list of the British Auchenorrhyncha (Hemiptera, Homoptera). Handbooks for the identification of British Insects, 2 (2c). Royal Entomological Society of London. Londres. 95 pp.

LindBerg, H., 1961. Hemiptera Insularum Madeirensium. Commentationes Biologicae, 24: 1-82.

Medina, V., Archelos, D., Llácer, G., Casanova, R., SÁnchez-CAPuchino, J.A., Martínez, A. \& GarriDO, A., 1981. Contribución al estudio de los cicadelidos (Homoptera, Cicadellidae) en las provincias de 
Valencia y Murcia. Anales del INIA. Serie Agrícola, 15: 157-179.

Medina, V., Garrido, A. \& Alfaro, A., 1982. Nota preliminar sobre cicadelidos (Homoptera: Cicadellidae) de los arrozales valencianos. Anales del INIA. Serie Agrícola, 19: 125-137.

NAST, J., 1972. Palaearctic Auchenorrhyncha (Homoptera). An annotated check list. Polish Scientific Publishers. Varsovia. 499 pp.

NAST, J., 1987. The Auchenorrhyncha (Homoptera) of Europe. Annales Zoologici, 40: 535-661.

Ossianilsson, F., 1981. The Auchenorrhyncha (Homoptera) of Fennoscandia and Denmark. Part 2. The families Cicadidae, Cercopidae, Membracidae, and Cicadellidae (excl. Deltocephalinae). Fauna Entomologica Scandinavica, vol 7. Part 2. Scandinavian Science Press Ltd. Copenhague. 223-593.

Ottosson, J.G. \& Anderson, J.M., 1983. Number, seasonality and feeding habits of insects attacking ferns in Britain - an ecological consideration. Journal of Animal Ecology, 52: 385-406.

QuARTAu, J.A. \& FAnÇONY, A.I., 1988. On Eupteryx capreola Lindberg and E. filicum (Newman) (Homoptera: Cicadellidae: Typhlocybidae) from Madeira and the Azores. Boletim do Museu Municipal do Funchal, 40: 93-103.

Remane, R., 1987. Zum Artenbestand der Zikaden (Homoptera: Auchenorrhyncha) auf dem Mainzer Sand. Mainzer Naturwissenschaft Archiv, 25: 273349.

Ribaut, H., 1936. Homoptères Auchènorhynches 1. (Typhlocybidae). Faune de France 31. Paul Le Chevalier et Fils. París. 228 pp.

Ruiz Castro, A., 1943. Dos tiflocíbidos nuevos en España que atacan a la vid y al pimiento. Boletín de Patología Vegetal y Entomología Agrícola, 12: 143189.

Ruiz Castro, A. \& MendizÁbal, M., 1939. La "roya colorada", producida por Empoasca libyca de Bergevin (Hem. Hom.), en los parrales de Almería. Boletín de Patología Vegetal y Entomología Agrícola, 8: 150-181.
SHIEMENZ, H. 1990. Beiträge zur Insektenfauna der DDR: Homoptera - Auchenorrhyncha (Cicadina) (Insecta). Teill III. Unterfamilie Typhlocybinae. Faunistische Abhandlugen Staatliches Museum für Tierkunde Dresden, 17:141-188.

STEWART, A.J.A., 1988. Patterns of host-plant utilization by leafhoppers in the genus Eupteryx (Hemiptera: Cicadellidae) in Britain. Journal of Natural History, 22: $357-379$.

Tutin, T.G., Heywood, V.H., Burges, N.A., Valentine, D.H., WAlters, S.M. \& WebB, D.A., (Eds.). 1964. Flora Europaea. Vol. 1. Lycopodiaceae to Platanaceae. Cambridge University Press. Oxford. 464 pp.

Tutin, T.G., Heywood, V.H., Burges, N.A., Valentine, D.H., Walters, S.M. \& WebB, D.A., (Eds.). 1968. Flora Europaea. Vol. 2. Rosaceae to Umbelliferae. Cambridge University Press. Oxford. 455 pp.

Tutin, T.G., Heywood, V.H., Burges, N.A., Moore, D.M., Valentine, D.H., Walters, S.M. \& WebB, D.A., (Eds.). 1980. Flora Europaea. Vol. 5. Alismataceae to Orchidaceae (Monocotyledones). Cambridge University Press. Oxford. 452 pp.

Vidano, C. \& Arzone, A., 1987a. Typhlocybinae of broadleaved trees and shrubs in Italy. 4. Fagaceae. Redia, 70: 171-189.

VidAno, C. \& ARzone, A., 1987b. Typhlocybinae of broadleaved trees and shrubs in Italy. 2. Betulaceae. Bollettino dell'Istituto di Entomologia Guido Grandi della Università di Bologna, 41: 257-267.

Vidano, C., Arzone, A. \& Arno, C.,1987. Host recognition and specificity of important European Typhlocybines. Proceedings of the $2^{\text {nd }}$ International Workshop on Leafhoppers and Planthoppers of Economic Importance, Provo, Utah, USA. 28 julio-1 agosto 1986. CIE. Londres: 283-292. 